\title{
Homology modeling and docking studies of ENPP4: a BCG activated tumoricidal macrophage protein
}

\author{
Dongmei Yan ${ }^{1 \dagger}$, Weiwei Han²', Zehua Dong ${ }^{3}$, Qihui Liu', Zheng Jin', Dong Chu', Yuan Tian', Jinpei Zhang ${ }^{1}$, \\ Dandan Song ${ }^{1}$, Dunhuang Wang ${ }^{1}$ and Xun Zhu ${ }^{1 *}$
}

\begin{abstract}
Background: The 3D structure and functions of ENPP4, a protein expressed on the surface of Bacillus CalmetteGuerin (BCG)-activated macrophages, are unknown. In this study, we analyzed the 3D structure of ENPP4 and determined its tumoricidal effects on MCA207 cells.

Results: Homology modeling showed that Arg305, Tyr341, Asn291, and Asn295 are important residues in substrate, adenosine triphosphate (ATP), binding. A molecular dynamics study was also carried out to study the stability of ENPP4 (including zinc atoms) as well as its ligand-enzyme complex. BCG increased ENPP4 expression in macrophages, and specific blocking of ENPP4 in BCG-activated macrophages (BAMs) significantly reduced their cytotoxicity against MCA207 cells.
\end{abstract}

Conclusions: These results indicate that zinc remains inside the ENPP4 protein, a BCG activated tumoricidal macrophage protein, throughout the simulation. Important information for the design of new inhibitors was obtained.

Keywords: Macrophages, ENPP4 protein, Homology modeling, Molecular docking, Anti-tumor activity

\section{Background}

Macrophages are the first line of defense of the human body against cancer development. Bacillus CalmetteGuerin (BCG) is a highly effective mediator that initiates the tumoricidal effect of macrophages through direct contact [1-5]. However, the mechanism of the antitumor effects of BCG remains unknown.

BCG can bind with TLR2 and TLR4 and upregulate certain proteins expressed on the macrophage surface or secreted to the microenvironment [6]. Activated macrophages can secrete large amounts of pro-inflammatory cytokines, such as tumor necrosis factor- $\alpha(\mathrm{TNF}-\alpha)$ and interlukine-12(IL-12), Nitric oxide(NO), reactive oxygen intermediates and highly express MHC-II and the costimulatory molecules CD80 and CD86 [7]. These molecules contribute to the tumoricidal effects of macrophages. Aside from these molecules [8], other membrane proteins

\footnotetext{
*Correspondence: zxunzhux@vip.sohu.com

${ }^{\dagger}$ Equal contributors

'Department of Immunology, College of basic Medical sciences, Jilin University, Xinmin Street 126\#, Changchun City, Jilin Province, China Full list of author information is available at the end of the article
}

involved in anti-tumor activities have been found to exist on the surface of BCG-activated macrophage (BAMs), as proven by the LS-LSM/MS technique.

Ectonucleotide pyrophosphatase-phosphodiesterases 4 (ENPP4) is an upregulated protein expressed on the BAM surface [2, 3]. However, its functions are incompletely understood. The nucleotide pyrophosphatase-phosphodiesterase or ectonucleotide pyrophosphatase-phosphodiesterase family includes eight members (NPP1-NPP8 or ENPP1-ENPP8) [9-11]. ENPP1-ENPP3 are composed of two N-terminal somatomedin B (SMB)-like domains (SMB1 and SMB2), a catalytic domain, and a nuclease-like domain (ENPP3 is monomeric). These phosphodiesterases are type- 2 transmembrane metalloenzymes and have a short intracellular $\mathrm{N}$-terminus, a single transmembrane region, and an extracellular domain that contains a catalytic site. By contrast, members ENPP4-ENPP7 consist of a single catalytic domain. Isoforms ENPP4 and ENPP5 are type-1 transmembrane proteins with a short intracellular C-terminus and a small extracellular region that contains only a phosphodiesterase motif. 
The related physiological and pathological roles of ENPP family members, including their regulation of extracellular pyrophosphate levels, cell motility, migration, angiogenesis, and tumor cell invasion, have recently become the focus of intensive research [12-15]. The isozyme ENPP1 catalyzes nucleotides that generate pyrophosphate, which can prevent excessive bone calcification. The subtype ENPP2 (or autotaxin) hydrolyzes lysophosphatidylcholine into lysophosphatidic acid and is involved in vasculature and neural tube formation and lymphocyte migration [12]. The enzyme ENPP3 (CD203) is a basophile marker [13] with a glycosylated type II transmembrane structure. ENPP5 has not been widely studied, but its closely related molecule, ENPP2, has been widely implicated in neoplasia and extensively studied in efforts to develop small-molecule inhibitors [14]. In fact, ENPP2 has been identified as a novel angiogenesis-associated gene [15]. The isoform ENPP6 has lysophospholipase-C activity for choline-containing glycerophosphodiesters, and the isoform NPP7 has alkaline sphingomyelinase activity and catalyzes lysophosphatidylcholine. No study on the structure and functions of ENPP8 has yet been reported. Although some studies have reported the structure of ENPP4, its 3D structure and related functions are largely unknown.

The present study aims to analyze the 3D structure of ENPP4 on the BAM surface through molecular modeling and study its tissue distribution and tumoricidal effects through immunohistochemistry.

\section{Results}

Homology modeling

The search for the best template for modeling ENPP4 was carried out using PSI-BLAST against PDB. ENPP1 (PDB code4GTX) [10] showed the best sequence similarity with ENPP4 (39 \%, Fig. 1). A phylogenetic tree of ENPP4 and its closest related sequences was constructed based on multiple sequence alignment (Fig. 2). This tree showed that ENPP4 has one clade, the ENPP4 subfamily. ENPP1 (PDB Id 4GTX) was selected as the template for ENPP4 modeling studies.

The initial model of ENPP4 was generated using a Swiss model. The initial model was refined by an energy minimization protocol in Gromacs 4.5.1. The final model was validated using four tools: Values of the rootmean square deviation (RMSD) from the initial structure were stable after approximately 2 ns (Additional file 1: Figure S1); such stabilization indicates that the trajectories have equilibrated. The final structure was further evaluated by Procheck. Ramachandran plot showed that the backbone dihedral angle distributions of all of the amino acid residues were $84.3 \%$ in the core region, $13.4 \%$ in the allowed region, and $1.3 \%$ in the generously allowed region, as presented in Table 1. Meanwhile, the template ENPP1 (PDB Id 4GTX) presented $88.7 \%$ in the core region and $11.3 \%$ in the allowed region. These results indicate that the backbone dihedral angles of the ENPP4 model are reasonably accurate. The ERRAT

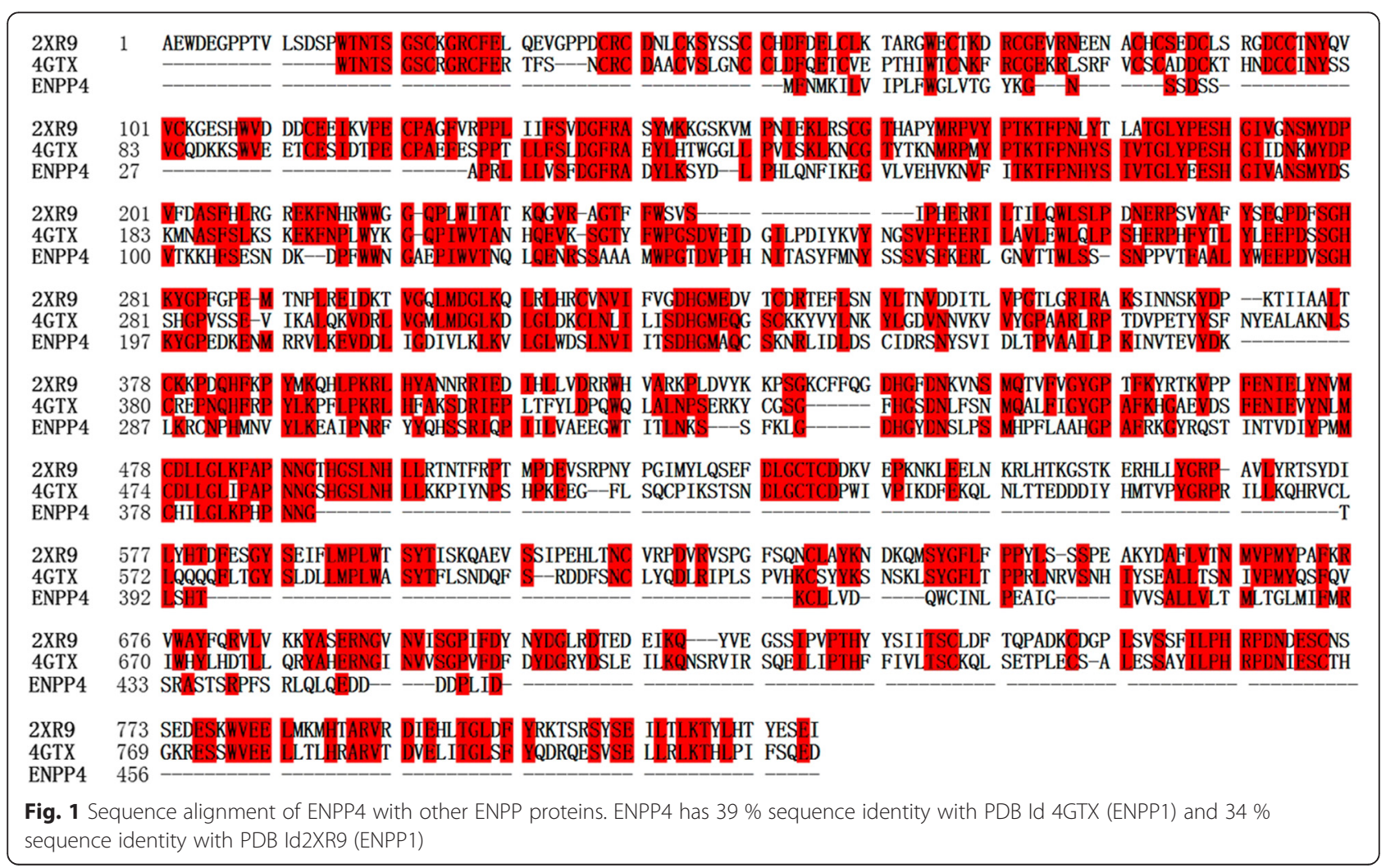




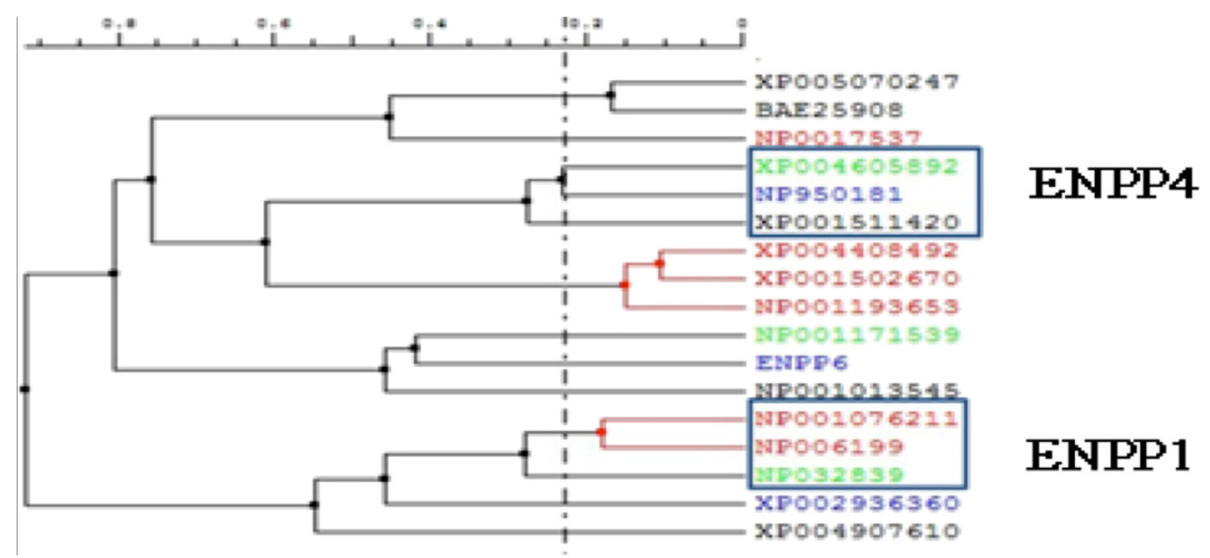

Fig. 2 A phylogenetic tree of ENPP4 and related families

score for ENPP4 was 86.36, whereas that for the template was 88.53 (Table 1); these scores are very similar. The final conformation with the lowest energy was chosen. Comparison of the model of the 3D structure of ENPP4 and the crystal structure of ENPP1 showed similar distributions of secondary structures (Fig. 3). The 3D structure supermposed between the ENPP1 and ENPP4 was $0.13 \AA$.

All of these findings indicate that the 3D structure of ENPP4 obtained by MD simulation is reasonable and can be used for further investigations.

\section{Docking study}

ENPP family proteins are membrane-bound glycoproteins. ENPP1 has been reported to cause human diseases characterized by ectopic calcification [16]. ENPP2 is a secreted lysophospholipase D that hydrolyzes lysophosphatidylcholine to produce lysophosphatidic acid (LPA), which, in turn, activates $\mathrm{G}$ protein-coupled receptors to evoke various cellular responses [17]. The five other mammalian ENPP proteins, ENPP3-ENPP7, have distinct substrate specificities and tissue distributions and thus participate in different biological processes. However, the 3D structure and biological functions of ENPP4 remain unknown.

ENPP1-ENPP3 are composed of two N-terminal SMBlike domains (SMB1 and SMB2), a catalytic domain, and a nuclease-like domain, whereas ENPP4-ENPP7 consist of a catalytic domain and lack the SMB-like and nuclease-

Table 1 The 3D models validation with Procheck, and Errat

\begin{tabular}{llc}
\hline Protein & Procheck & Errat \\
\hline ENPP4 & $\begin{array}{l}\text { Ramachandran plot: 84.3 \% } \\
\text { core 13.4 \% allow 1.3\% gener }\end{array}$ & 86.36 \\
ENPP1 (PDB Id 4GTX) & $88.7 \%$ core 11.3\% allow 0.0 \% gener & 88.53 \\
\hline
\end{tabular}

like domains [16]. To determine the binding site between the protein and the substrate, the cavity volume was estimated by CASTp as a function of the radius of the probe sphere. A probe radius of $1.4 \mathrm{~A}$ outlined a cavity of 2237.4 $\AA^{3}$ for ENPP4 (Additional file 2: Figure S2A) and a cavity of $1177.7 \AA^{3}$ for ENPP1 (Additional file 2: Figure S2B). The active pockets of ENPP4 and the template were similar. The active residues in ENPP1 were Asp358, His362, His517, Asp200, Thr238, Asp405, and His406. In ENPP4, Asp192, His196, His339 Asp37, Thr73, Asp240, and His241 were located in the catalytic domain. The reconstructed structure of ENPP4 reveals that the insertion loop (residues 139-158) participates in the formation of the substrate-binding pocket. In particular, M155 and S158 are located on the protein surface, whereas P140 and W138 are located at the bottom of the active pocket. The different binding pockets observed may influence the substrate specificities of the two proteins.

The substrate, ATP, was docked in ENPP4. Figure 4a shows the binding pose of ATP in ENPP4. The substrate is located in the catalytic cleft and fits the binding pocket well. The interactions of ATP in the active cleft of ENPP4 are shown in Fig. 4b. Five hydrogen bonds were observed between ATP and ENPP4. Figure 4b shows that Arg305 forms two hydrogen bonds with ATP. The $\mathrm{OH}$ group of Tyr341 produces a hydrogen bond with ATP, and the adenosine group of ATP forms two hydrogen bonds with Asn291 and Asn295. Asp342, Tyr341, Arg305, Tyr297, Met294, Pro292, Asn291, Asn295, Arg289, and Cys290 show electrostatic interactions with ENPP4, whereas Pro303, Phe306, Val320, Glu322, His293, and Lys288 show van der Waals interactions with ENPP4. These results may be helpful in designing future ATP analogue inhibitors. The ligand was mainly stabilized by the hydrogen bonds determined during our MD simulation. Arg305, Tyr341, Asn291, and Asn295 allowed hydrogen-bonding with ENPP4. 


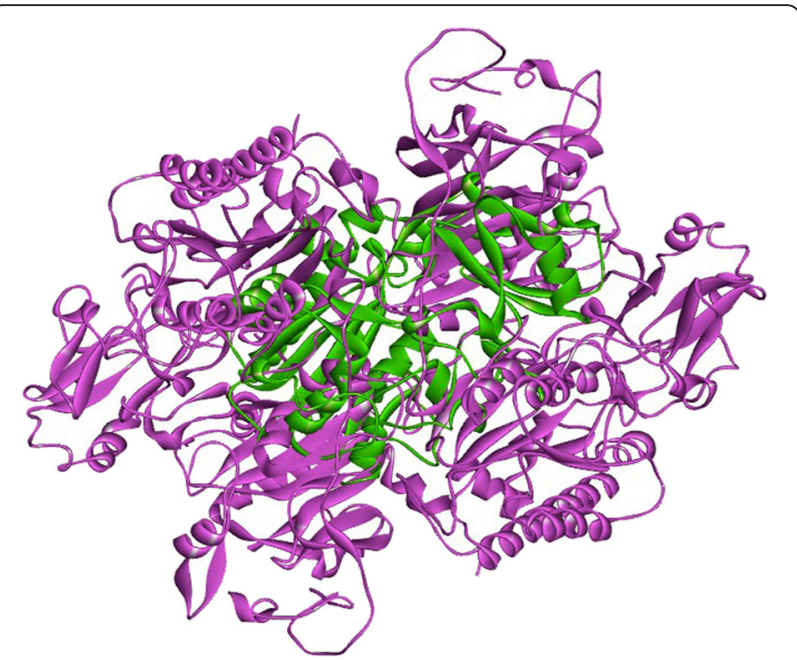

Fig. 3 Overlay similarity between the ENPP4 (green) and the template ENPP1 (purple)

Among these residues, only Tyr431, which is located near the His339-coordinated $\mathrm{Zn}^{2+}$ in the ENPP4 family (Fig. 1).

\section{Construction of pET-28a-ENPP4 and expression of recombined ENPP4 protein and its polyantibody}

The DNA fragment with an expected size of $750 \mathrm{bp}$ was obtained by PCR and enzyme digestion with EcoRI and

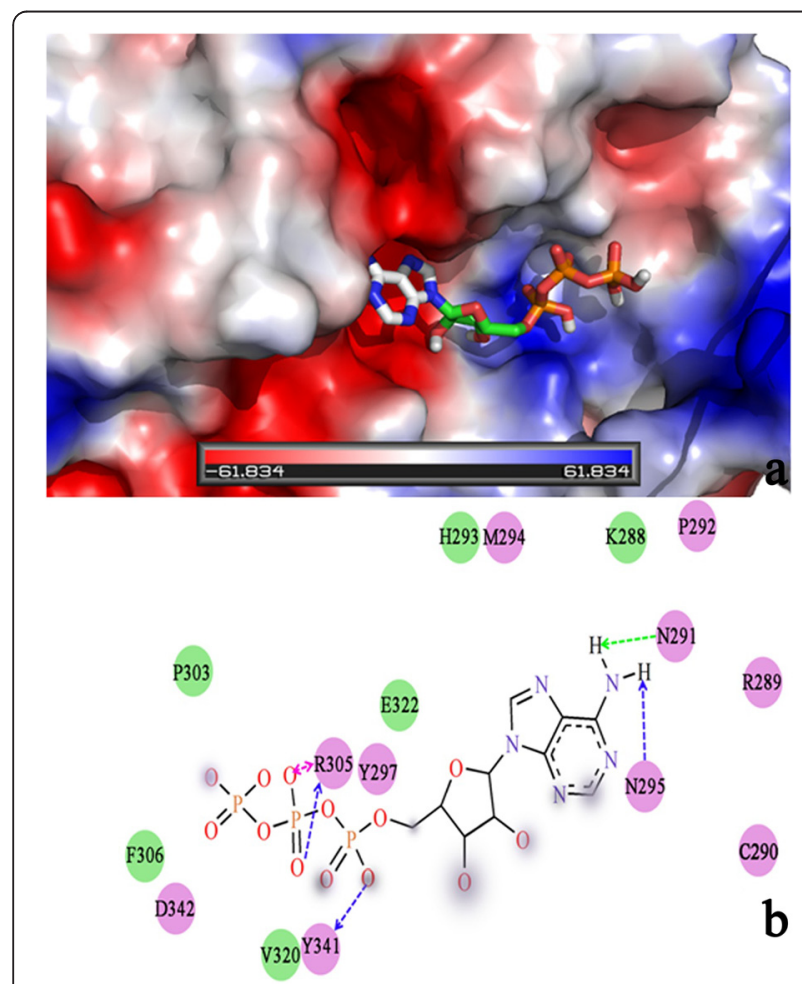

Fig. 4 a: the substrate in the active site. $\mathbf{b}$ : ENPP4 -ATP interactions
Xhol, which demonstrates that the correct pET-28aENPP4 plasmids were constructed (Fig. 5a and b). After the pET-28a-ENPP4 recombinant plasmid was transformed in $E$. coli Rosetta (DE3), the expression of ENPP4 was detected and shown in Fig. 5c and d, (32 KD MW protein). A large amount of recombinant ENPP4 protein was obtained in insoluble form. The resulting protein showed a purity of over $90 \%$, as determined by SDS-PAGE (Fig. 5c). Polyclonal antibodies were produced in rabbits. Figure $5 \mathrm{~d}$ shows the high specificity of the anti-ENPP4 polyclonal antibodies for binding to ENPP4, as determined by Western blot detection.

\section{Expression of ENPP4 in tissues}

The expression of ENPP4 was detected in 12 tissue samples from a normal female C57BL/6 mouse. ENPP4 was abundantly expressed in the spleen, stomach, and ovary (Fig. 6). No expression was observed in the brain, lung, kidney, thymus, liver, heart, uterus, and intestine. This result indicates that ENPP4 is involved in biological pathways related to immunity and reproduction.

\section{Tumoricidal activity of ENPP4 in BAMs}

To study the contact-dependent tumoricidal activity of ENPP4, cytotoxicity assays were carried out using paraformaldehyde-fixed macrophages. BAMs showed prominent cytotoxicity against MCA207 cells and this cytotoxic activities may be downregulated by blocking ENPP4 (Fig. 7a). The negative control did not exhibit cytotoxic effects. These results demonstrate that ENPP4 may be an essential functional molecule in the BAMmediated killing of MCA207 cells. Furthermore, cytotoxicity experiment results showed that ENPP4 protein exerts direct tumoricidal activities against MCA207 cells (Fig. 7b).

\section{Discussion}

MD simulation was employed to assess the stability of the homology model and the ligand-enzyme complex. In the homology model, we focused on the catalytic site, particularly on the amino acids that coordinate $\mathrm{Zn}^{2+}$ and the interactions between the metal and the enzyme. MD simulation was used to analyze the ENPP4-ATP complex and identify the possible bioactive conformation of the molecule. The conformation of ATP obtained from molecular docking and the ENPP4 model were used as the starting point for the simulation. The ligand was mainly stabilized by the hydrogen bonds determined during our MD simulation. Among Arg305, Tyr341, Asn291, and Asn295, only Tyr431 was conserved in the ENPP4 family. These observations suggest that this specific residue is necessary for the molecular evolution of the ENPP family. The plot shows the distance between the $\mathrm{H}$ in the oxygen of Tyr341 and ATP. Interestingly, the stable formation of 


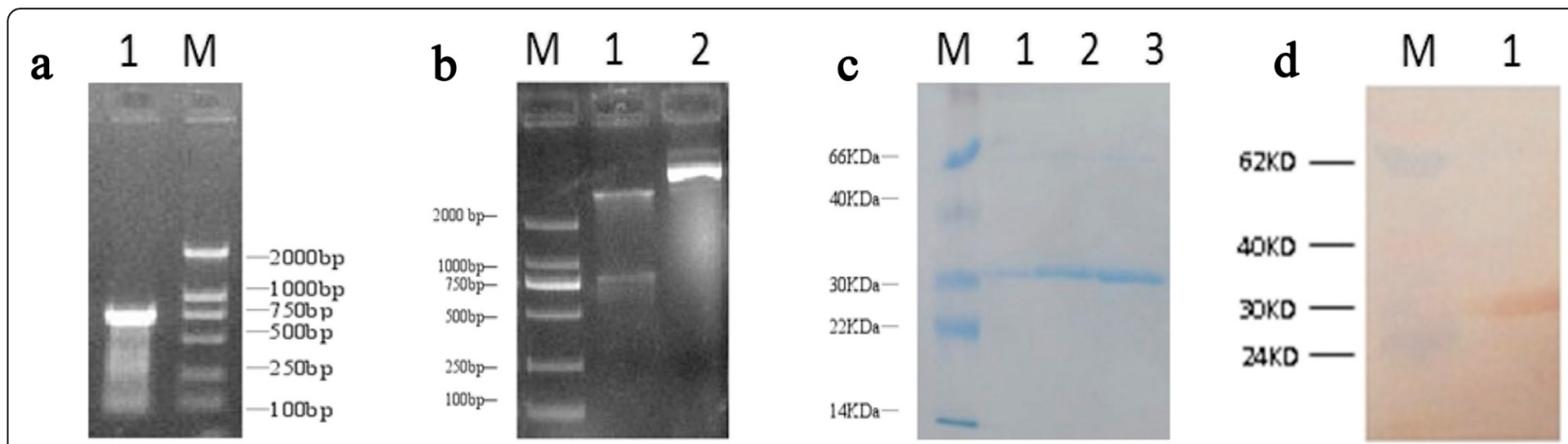

Fig. 5 a Analysis of ENPP4 CDNA sequence amplified by RT-PCR. b Enzyme restriction assay with restriction enzymes EcoRI and Xhol of pET-28aENPP4 vector construction. Lanes 1 shows the digested plasmids with its expected sizes released from constructed DNA-vectors. Lanes 2 shows the non-digested plasmids. c SDS gel electrophoretic patterns of recombinant ENPP4 after purification. Lanes 1-3: different concentration of ENPP4 protein stained by Coomassie blue. $\mathbf{d}$ PVDF membrane of western blotting assay of ENPP4 purified protein (32KD)

a hydrogen bond between the ligand and Asp141 was observed during the $10 \mathrm{~ns}$ MD simulation. This finding suggests that the residue of the catalytic site can stabilize interactions between $\mathrm{OH}$ and ATP; however, other studies featuring longer simulations are necessary to support this idea.
Mycobacterium bovis BCG is the most widely used vaccine in the world. BCG generates a local immunological reaction that activates immune cells, including polymorphonuclear and mononuclear cells, in bladder tumors after BCG therapy [12]. The ENPP family has been reported to be involved in various pathologies,
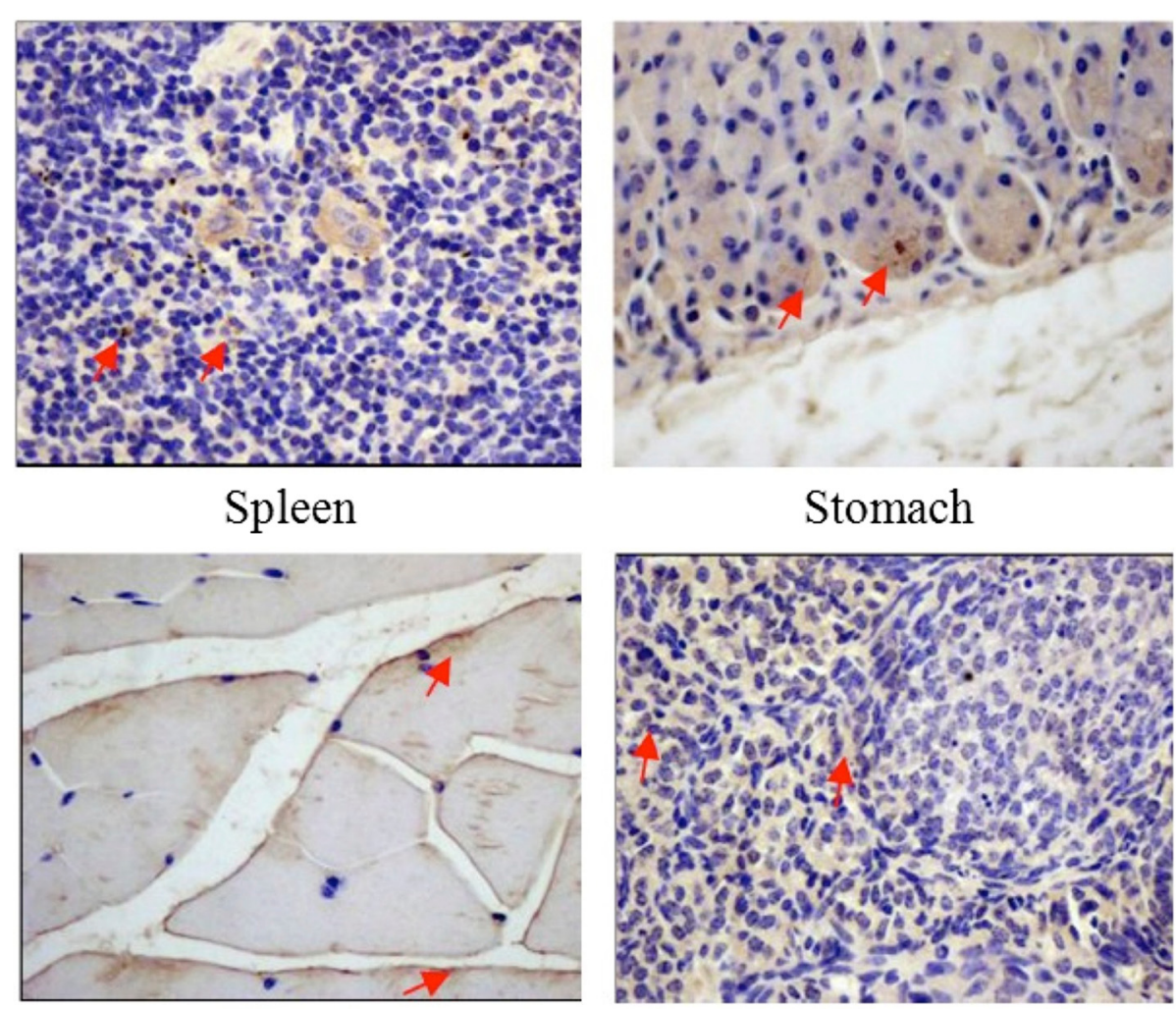

Muscle

Stomach

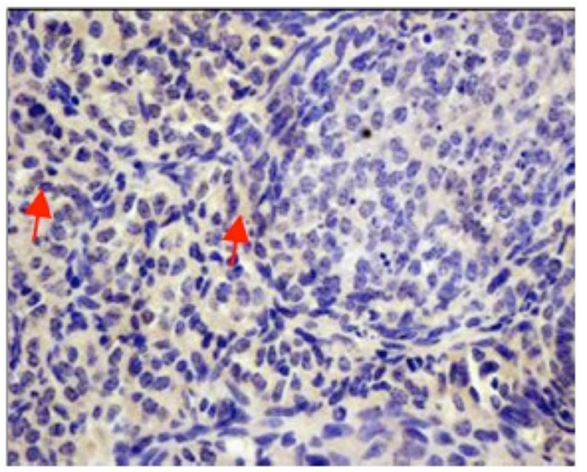

Ovary

Fig. 6 The expression of ENPP4 in different tissues, red arrow show abundant expression. Scoring was completed by a specialist pathologist and a scientist who were blinded to the pathologic information $(\times 400)$ 

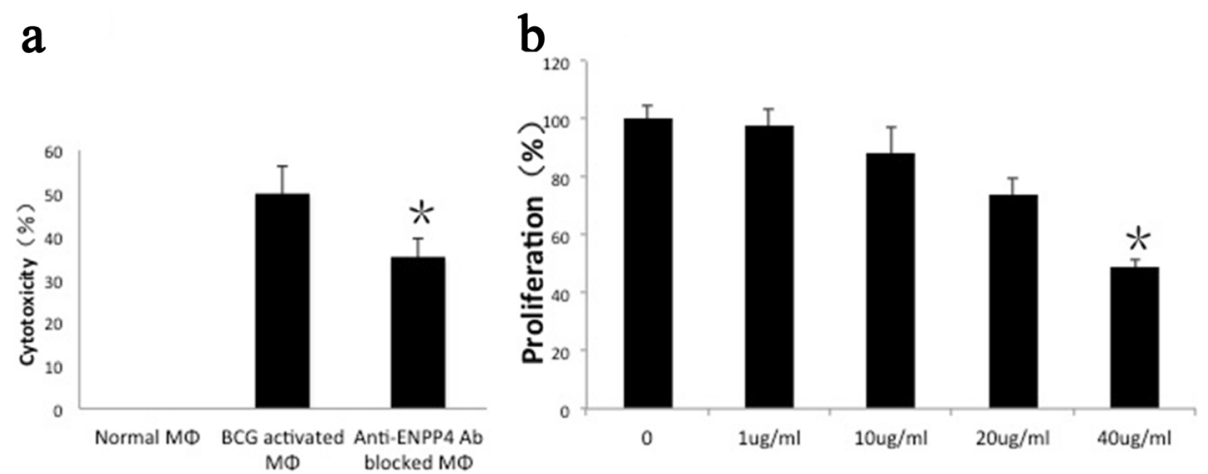

Fig. 7 ENPP4 has tumoricidal activity against MCA207 cells. a Antibodies against ENPP4 influenced the tumoricidal activity of macrophages. Negative control cells exhibited no cytotoxic activity, whereas BCG-activated macrophages exhibited a cytotoxicity of 50 \%. Blocking ENPP4 on BCG-activated macrophages decreased the cytotoxicity to $35.1 \%$. ${ }^{*}, P<0.05$ compared with positive control. $\mathbf{b}$ ENPP4 protein inhibited the proliferation of MCA207 cells in a dose-dependent manner. ${ }^{*}, P<0.05$ compared with negative control

including tumor progression and inflammation. ENPP2 is a secreted lysophospholipase $\mathrm{D}$ that generates the lipid mediator LPA, a mitogen, and a known chemoattractant for many cell types [18]. We have certified that the expression of ENPP4 is upregulated by BCG (Additonal file 3: Figure S3). Blocking ENPP4 on BAM significantly downregulates the anti-tumor activity of the cell, which demonstrates that ENPP4 has potential tumoricidal activity.

ENPP4 showed a catalytic domain in Asp192, His196, His339 Asp37, Thr73, Asp240, and His241, which suggests that the ENPP4 may affect some receptor such as ATP receptor or insulin receptor on the surface of tumor cells to reduce their proliferation by indirectly or directly contact, receptively $[19,20]$. On the one hand, ENPP4 may catalize the extracellular ATP released from tumor cells and reduce the binding between ATP and ATP receptor $[19,21]$, on the other hand, ENPP4 may contact the insulin receptor and inhibit the insulin receptor activity [20, 22].

Altergether, ENPP4 may thus be targeted as a therapeutic molecule for treating tumors. To explore the therapeutic potential of such a strategy, more detailed knowledge of the functions of ENPP4 and its ligand in tumor cells is needed.

\section{Conclusion}

Our findings provide novel insights into the structure of ENPP4 and help researchers better understand its diverse cellular functions.

\section{Methods}

All experiments conform to Jilin University guidelines on the ethical use of animals and were approved by the Institutional Animal Care and Use Committee. The mice used were C57BL/6 (wild-type, WT).

\section{Homology modeling}

The amino acid sequence of the target protein, ENPP4, was obtained from the National Center for Biotechnology Information NCBI (http://www.ncbi.nlm.nih.gov/) (GenBank: NP_950181; template protein: ENPP1 (PDB Id 4GTX) [16]. The BLAST search algorithm was used for an online search (http://www.ncbi.nlm.nih.gov). A modeler module was employed to build the 3D structure of the protein. Modeling was then carried out using Gromacs 4.5.1 software [23] with the Gromos53a6 allatom force field. The temperatures were kept constant at $\mathrm{T}=25{ }^{\circ} \mathrm{C}$ by coupling to a Berendsen thermostat with a coupling time of $\mathrm{T}=0.1 \mathrm{ps}$. The protein was solvated using a box of TIP3P [24] water molecules extending at least $10 \AA$ away from the boundary of any protein atom with an integration step of 2 fs. Non-bonded interactions were calculated using a cutoff of $10 \AA$. Long-range electrostatic interactions were calculated by Particle-Mesh Ewald summation with a grid spacing of $1.2 \AA$ and cubic interpolation. After 1000 steps of steepest descent energy minimization, the solvent and ions were equilibrated by 0.5 ns molecular dynamics (MD) simulation, with the heavy protein atoms subjected to harmonic constraints under a force constant of $\mathrm{k}=1000 \mathrm{kcal} /$ $\left(\mathrm{mol}^{-1} \cdot \mathrm{nm}^{-2}\right)$. Finally, a production run was carried out for $10 \mathrm{~ns}$, and the coordinates of all atoms at each picosecond were stored for further analysis. In our studies, CASTp (http://cast.engr.uic.edu/cast/) [25] was used to identify all of the cavities associated with the model and the template and measure their volumes. The results obtained were further used for proteinligand docking investigations.

\section{Docking study}

The software AutoDock Vina [26, 27] was applied for docking studies. The 3D structures of the substrate were 
downloaded from the Chemspider database. The target used in our study was the 3D structure of ENPP4. The grid size for docking measured $36 \times 36 \times 36 \AA^{3}$.

\section{Cloning of ENPP4 gene in the expression vector pET-28a}

To obtain a mouse peritoneal macrophage cell preparation, female C57BL/6 mice, 10 week of age, were immunized intraperitoneally (i.p.) with $4 \mathrm{mg}$ of BCG (Chengdu Institute of Biological Products) three times on days 2,10 , and 12 . Three days after the last i.p. injection, the mice were sacrificed and peritoneal cells were collected. Adherent macrophages were collected to prepare the total RNA and CDNA of ENPP4 by RTPCR $[2,3]$. Briefly, PCR reactions were performed using self-designed primer pairs: EF (5'-CGGGAATTCTCAGCACCTCGGTTACTT-3') and ER (5'-AAACTCGAGAAGAATCGCAGCCACAGG-3'). All amplification reactions consisted of an initial denaturation step at $94{ }^{\circ} \mathrm{C}$ for $5 \mathrm{~min}, 30$ cycles of $94{ }^{\circ} \mathrm{C}$ denaturation for $1 \mathrm{~min}, 57^{\circ} \mathrm{C}$ annealing for $1 \mathrm{~min}$, and $72{ }^{\circ} \mathrm{C}$ extension for $2 \mathrm{~min}$, followed by a final extension at $72{ }^{\circ} \mathrm{C}$ for 10 min using 2 units of Taq DNA polymerase. The cDNA of ENPP4 was then cloned to pET-28a to obtain the pET-28a-ENPP4 plasmid.

\section{Expression and purification of recombinant ENPP4 protein in Escherichia coli}

To determine the expression of the recombinant ENPP4 protein, Escherichia coli Rosetta (DE3) (Invitrogen) transformed with pET-28a-ENPP4 plasmid DNA was inoculated in a tube containing $10 \mathrm{~mL}$ of LB medium supplemented with kanamycin and chloromycetin. The tube was allowed to culture overnight at $37^{\circ} \mathrm{C}$ in a shaking incubator $(180 \mathrm{rpm})$ until the culture reached an OD of 0.6 when read at a wavelength of $600 \mathrm{~nm}$. Protein expression was induced by the addition of $0.2,0.4,0.6$, and $0.8 \mathrm{mM}$ isopropyl $\beta \mathrm{D}-1$-thiogalactopyranoside. After $6 \mathrm{~h}$ of induction at $37^{\circ} \mathrm{C}$, the cells were collected by centrifugation for sodium dodecyl sulfate-polyacrylamide gel electrophoresis (SDS-PAGE).

To obtain the recombinant ENPP4 protein, bacterial cells were harvested by centrifugation at $4{ }^{\circ} \mathrm{C}$ and resuspended in PBS buffer. The bacterial pellets were then mixed with $10 \mathrm{mmol} / \mathrm{L} \mathrm{MgSO} 4,0.01 \mathrm{mg} / \mathrm{mL}$ DNaseI, and $0.1 \mathrm{mg} / \mathrm{mL}$ lysis enzyme and left at $4{ }^{\circ} \mathrm{C}$ for $20 \mathrm{~min}$. After centrifugation, the bacterial pellets were loaded with ice-cold lysis buffer $(50 \mathrm{mmol} / \mathrm{L}$ Tris$\mathrm{Cl}, 0.1 \mathrm{~mol} / \mathrm{L} \mathrm{NaCl}, 5 \mathrm{mmol} / \mathrm{L}$ EDTA, $0.1 \% \mathrm{NaN}_{3}$, $0.5 \%$ Triton X-100, pH 6.8) and sonicated at $1000 \mathrm{~W}$ for $5 \mathrm{~s}$ with $10 \mathrm{~s}$ intervals for 160 cycles. The supernatant was discarded, and the pellet (inclusion bodies) containing the recombinant ENPP4 protein was subjected to dilute renaturation and dialysis renaturation and concentration. The purity of the extracted protein was detected by SDS-PAGE.

\section{Preparation of polyclonal antibodies and Western blot analysis}

Purified recombitant protein was used as the immunogen. Approximately $100 \mu \mathrm{g}$ of the fusion protein emulsified in complete Freund's adjuvant was administered during initial inoculation, and $50 \mu \mathrm{g}$ of fusion protein emulsified in incomplete Freund's adjuvant was given after 2 weeks. This procedure was repeated 5 times at 1 week intervals. Final rabbit serum was harvested after immunization.

ENPP4 peptides were separated by SDS-PAGE and electrotransferred onto the PVDF membrane. The membrane was blocked with $3 \%$ bovine serum albumin in PBS for $2 \mathrm{~h}$ at room temperature. Subsequently, antiENPP4 antibodies were added at a dilution of 1:1000 and incubated at room temperature for $1 \mathrm{~h}$. After washing thrice for $15 \mathrm{~min}$, IgG goat-anti-rabbit-HRP was added and incubated for $1 \mathrm{~h}$ at room temperature. After a final washing, the resulting signals were detected using DAB (3,3-diaminobenzidine tetrahydrochloride).

Immunohistochemistry for ENPP4 detection in mouse tissues Various mouse tissues (including heart, liver, kidney, spleen, muscle, lung, intestine, uterus, and ovary) were formalin-fixed at $4{ }^{\circ} \mathrm{C}$ overnight and then embedded in paraffin and sectioned for histology. Immunohistochemical staining of serial sections was performed to detect the expression and distribution of ENPP4. All samples were incubated in $0.3 \% \mathrm{H}_{2} \mathrm{O}_{2}$ for $10 \mathrm{~min}$ at room temperature to inactivate endogenous peroxidase. The paraffinized sections were then incubated with antiENPP4 primary antibody for $1 \mathrm{~h}$ at room temperature. After washing, the sections were incubated with peroxidase-conjugated goat anti rabbit IgG for $40 \mathrm{~min}$. After a final washing, the resulting signals were detected using $\mathrm{DAB}$ and photographed with a microscope.

\section{Tumoricidal effect of ENPP4 on MCA207 cells}

BAM-mediated tumor cytotoxicity was determined with and without anti-ENPP4 antibodies. Peritoneal macrophages were harvested from mice and fixed with $1 \%$ paraformaldehyde for $30 \mathrm{~min}$ at room temperature. Afterward, $6 \times 10^{5}$ cells were incubated for $2 \mathrm{~h}$ with $5 \mu \mathrm{L}$ of anti-ENPP4 serum (previously prepared by our research group) and $5 \mu \mathrm{L}$ of control serum (from preimmunized rabbit). The blocked cells were then washed twice with RPMI 1640 medium, seeded into a 96-well flat-bottom plate containing $1 \times 10^{4}$ MCA207 target cells/well, and incubated for $48 \mathrm{~h}$. The number of viable tumor cells was determined by MTT $(5 \mathrm{mg} / \mathrm{mL}$, Sigma) assay. The absorbance at $570 \mathrm{~nm}$ was recorded directly using a microplate reader (Model 550, Bio-RAD).

Purified ENPP4 protein (prepared by our team) was added to MCA207 $\left(1 \times 10^{4}\right.$ cells, cultured in 96-well 
plates) at concentrations of $1,10,20$, and $40 \mu \mathrm{g} / \mathrm{mL}$, and the cells were incubated for $48 \mathrm{~h}$ at $37{ }^{\circ} \mathrm{C}$ under a $5 \%$ $\mathrm{CO}_{2}$ atmosphere. The proliferation levels of MCA207 were detected by MTT assay.

\section{Statistical analysis}

Each experiment was repeated at least three times. Data were expressed as mean \pm SEM and analyzed by one-way analysis of variance. $P$ values $<0.05$ (95\% confidence level) were considered statistically significant.

\section{Additional files}

Additional file 1: Figure S1. The RMSD of ENPP4 during the 10 ns MD simulation. (JPG $172 \mathrm{~kb}$ )

Additional file 2: Figure S2 a: The binding pocked of ENPP4 (calculated by CASTp, $2237.4 \AA 3$ ); b: The binding pocket of ENPP1 (PDB Id 4GTX) (calculated by CASTp,. $1177.7 \AA 3$ ). (JPG 223 kb)

Additional file 3: Figure S3. BCG increased the expression of ENPP4 in the macrophages. (JPG $22 \mathrm{~kb}$ )

\section{Competing interests}

The authors declare no competing financial interests.

\section{Authors' contributions}

XZ and DMY designed the study, WWH performed the bioinformation sequence and structural analysis, and others performed the experiments. DMY and WWH drafted the manuscript. All authors read and approved the final version of the manuscript.

\section{Acknowledgements}

This work was funded by a grant from the National Program On Key Basic Research Project (973 Program No. 2012CB721003) and National Natural Science Foundation of China(81571530).

\section{Author details}

'Department of Immunology, College of basic Medical sciences, Jilin University, Xinmin Street 126\#, Changchun City, Jilin Province, China. ${ }^{2}$ Key Laboratory for Molecular Enzymology and Engineering, Ministry of Education, Jilin University, Qianjin Street 2699\#, Changchun City, Jilin Province, China. ${ }^{3}$ Intensive Care Unit, The Affiliated Hospital of Qingdao University, Jiangsu Road 16\#, Qingdao, China.

Received: 6 October 2015 Accepted: 21 January 2016

Published online: 28 January 2016

\section{References}

1. Quiding-Järbrink M, Raghavan S, Sundquist M. Enhanced M1 macrophage polarization in human helicobacter pylori-associated atrophic gastritis and in vaccinated mice. PLoS One. 2010:5(11):e15018.

2. Tsung K, Dolan JP, Tsung YL, Norton JA. Macrophages as effector cells in interleukin 12-induced T cell-dependent tumor rejection. Cancer Res. 2002; 62(17):5069-75.

3. Zhang L, Lun Y, Yan D, Yu L, Ma W, Du B, et al. Proteomic analysis of macrophages: A new way to identify novel cell-surface antigens. J Immunol Methods. 2007:321(1-2):80-5.

4. Liu X, Dowell AC, Patel P, Viney RP, Foster MC, Porfiri E, et al. Cytokines as effectors and predictors of responses in the treatment of bladder cancer by bacillus Calmette-Guérin. Future Oncol. 2014;10(8):1443-56.

5. Lodillinsky C, Langle Y, Guionet A, Góngora A, Baldi A, Sandes EO, et al. Bacillus calmette guerin induces fibroblast activation both directly and through Macrophages in a mouse bladder cancer model. PLoS One. 2010; 5(10):e13571.

6. Arko-Mensah J, Julián E, Singh M, Fernández C. TLR2 but not TLR4 signalling is critically involved in the inhibition of IFN-gamma-induced killing of mycobacteria by murine macrophages. Scand J Immunol. 2007;65(2):148-57.
7. Sica A, Invernizzi P, Mantovani A. Macrophage plas- ticity and polarization in liver homeostasis and pathology. Hepatology. 2014;59(5):2034-42.

8. Zhang L, Zhu H, Lun Y, Yan D, Yu L, Du B, et al. Proteomic analysis of macrophages: a potential way to identify novel proteins associated with activation of macrophages for tumor cell killing. Cell Mol Immunol. 2007; 4(5):359-67.

9. Nowak-Machen M, Lange M, Exley M, Wu S, Usheva A, Robson SC. Lysophosphatidic acid generation by pulmonary NKT cell ENPP-2/autotaxin exacerbates hyperoxic lung injury. Purinergic Signal. 2015;11(4):455-61.

10. Nakanaga K, Hama K, Aoki J. Autotaxin: an LPA producing enzyme with diverse functions. J Biochem. 2010;148(1):13-24.

11. Hausmann J, Perrakis A, Moolenaar WH. Structure-function relationships of autotaxin, a secreted lysophospholipase D. Adv Biol Regul. 2013;53(1):112-7.

12. Cheng HY, Dong A, Panchatcharam M, Mueller P, Yang F, Li Z, et al. Lysophosphatidic acid signaling protects pulmonary vasculature from hypoxiainduced remodeling. Arterioscler Thromb Vasc Biol. 2012;32(1):24-32.

13. Thiel A, Kesselring R, Pries R, Wittkopf N, Puzik A, Wollenberg B. Plasmacytoid dendritic cell subpopulations in head and neck squamous cell carcinoma. Oncol Rep. 2011;26(3):615-20.

14. Liu S, Murph M, Panupinthu N, Mills GB. ATX-LPA receptor axis in inflammation and cancer. Cell Cycle. 2009;8(22):3695-701

15. Smith SJ, Tilly H, Ward JH, Macarthur DC, Lowe J, Coyle B, et al. CD105 (Endoglin) exerts prognostic effects via its role in the microvascular niche of paediatric high grade glioma. Acta Neuropathol. 2012;124(1):99-110.

16. Kato K, Nishimasu H, Okudaira S, Mihara E, Ishitani R, Takagi J, et al. Crystal structure of Enpp1, an extracellular glycoprotein involved in bone mineralization and insulin signaling. Proc Natl Acad Sci U S A. 2012;109(42): 16876-81.

17. Umezu-Goto M, Kishi Y, Taira A, Hama K, Dohmae N, Takio K, et al. Autotaxin has lysophospholipase D activity leading to tumor cell growth and motility by lysophosphatidic acid production. J Cell Biol. 2002;158:227-33.

18. Hausmann J, Kamtekar S, Christodoulou E, Day JE, Wu T, Fulkerson Z, et al. Structural basis of substrate discrimination and integrin binding by autotaxin. Nat Struct Mol Biol. 2011;18(2):198-204.

19. Chin CN, Dallas-Yang Q, Liu F, Ho T, Ellsworth K, Fischer P, et al. Evidence that inhibition of insulin receptor signaling activity by PC-1/ENPP1 is dependent on its enzyme activity. Eur J Pharmacol. 2009:606(1-3):17-24.

20. Salisbury TB, Tomblin JK. Insulin/Insulin-like growth factors in cancer: new roles for the aryl hydrocarbon receptor, tumor resistance mechanisms, and new blocking strategies. Front Endocrinol (Lausanne). 2015;6:12.

21. Giannuzzo A, Pedersen SF, Novak I. The P2X7 receptor regulates cell survival, migration and invasion of pancreatic ductal adenocarcinoma cells. Mol Cancer. 2015;14(1):203

22. Gallagher EJ, Alikhani N, Tobin-Hess A, Blank J, Buffin NJ, Zelenko Z, et al. Insulin receptor phosphorylation by endogenous insulin or the insulin analog AspB10 promotes mammary tumor growth independent of the IGFreceptor. Diabetes. 2013;62(10):3553-60

23. Gruber CC, Pleiss J. Systematic benchmarking of large molecular dynamics simulations employing GROMACS on massive multiprocessing facilities. J Comput Chem. 2011:32(4):600-6.

24. Jorgensen WL, Chandrasekhar J, Madurs J, Impey RW, Klein ML. Comparison of simple potential functions for simulating liquid water. J Chem Phys. 1983; 79:926-35.

25. Dundas J, Zheng O, Tseng J, Binkowski A, Turpaz Y, Liang Y. CASTp: computed atlas of surface topography of proteins with structural and topographical mapping of functionally annotated residues. Nucleic Acids Res. 2006:34:W116-8.

26. Seeliger D, de Groot BL. Ligand docking and binding site analysis with PyMOL and AutodockNina. J Comput Aided Mol Des. 2010:24(5):417-22.

27. Trott O, Olson A. AutoDock Vina: improving the speed and accuracy of docking with a new scoring function, efficient optimization, and multithreading. J Comput Chem. 2010;31(2):455-61. 